\title{
Acute carotid sinus stimulation in uncontrolled hypertensive patients
}

\author{
Márcio Galindo Kiuchi*1 and Shaojie Chen ${ }^{2}$ \\ ${ }^{1}$ Division of Cardiac Surgery and Artificial Cardiac Stimulation, Department of Medicine, Hospital e Clínica São Gonçalo, Brazil \\ ${ }^{2}$ Department of Cardiology, Shanghai First People's Hospital, Shanghai Jiao Tong University School of Medicine, Shanghai, China
}

Extended electric stimulation of the carotid baroreceptors through its modulatory effect on afferent signaling is an attractive target for the treatment of uncontrolled hypertension. Baroreflex sensitivity is usually distressed in hypertension and be unsuccessful to keep blood pressure (BP) at proper levels through its fast-negative feedback loop, in which a raised BP reflexively origins the heart rate to fall and BP to drop. In an initial proof-of-concept study, BAT has been found to exert considerable BP-lowering properties using the CVRx Rheos System (CVRx, Minneapolis, MN) (DEBuT-HT [Device Based Therapy in Hypertension Trial) comprising implantable electrodes connected to a subcutaneously placed stimulator [1,2]. Implantation of the first-generation device was related to serious procedure-related adverse events, and the short-term battery life restricted its utility. Next-generation devices could essentially overawe these issues and implantation of the Barostim neo (CVRx) was associated with a significant BP decline at 3 and 6 months of follow-up [3]. A recent study in 28 patients [4] exposed further evidence of the positive effects reached by BAT in patients who continued hypertensive despite renal sympathetic denervation performed 5 months earlier. While the BAT device is typically initiated 2 to 4 weeks after surgical implantation to permit the site to heal, immediate activation of BAT in this specific situation created a quick, significant, and sustained reduction In BP. Even though a device that needs surgical placement of electrodes maybe impractical in an emergency condition, the concept of electrical stimulation of the baroreceptors as a means of accomplishing speedy and sustained BP reduction is fascinating and highlights the prospective of this methodology. Further enhancement of the technology and longerterm follow-up of treated patients will support to delineate the clinical value of BAT as an alternative treatment for resistant hypertension.

The well-recognized association between augmented baroreceptors response and parasympathetic activation as an important contributor to the pathophysiology of hypertension led to the initiation of studies investigating the feasibility of a therapeutic intervention aimed at selectively targeting arterial baroreceptors located in the carotid sinus. This transversal study involved 18 uncontrolled hypertensive patients, complaining of pre-syncope. The study was piloted in agreement with the Helsinki declaration and approved by the ethics committee of our institution. All patients signed the informed consent term before inclusion. This study was conducted at the Hospital e Clínica São Gonçalo, Rio de Janeiro, Brazil. Patients were recruited from January to August 2016 from the Arrhythmias and Artificial Cardiac Pacing Service of the same hospital. Patients with the combination of the following criteria were consecutively enrolled: (i) mean 24-hour systolic ambulatory blood pressure measurements (ABPM) $\geq 130 / \geq 80$ $\mathrm{mmHg}$ despite treatment with non-pharmacological measures and use of at least three antihypertensive drugs (including a diuretic) on maximally tolerated doses or confirmed intolerance to medications; (ii) CKD: glomerular filtration rate estimated by the CKD-EPI (Chronic Kidney Disease Epidemiology Collaboration) equation, eGFR, [5] $>60 \mathrm{~mL} / \mathrm{min} / 1.73 \mathrm{~m}^{2}$ without microalbuminuria; (iii) age from 18 to 80 years; (iv) complaining of pre-syncope symptoms; (v) negative tilt-table testing and normal carotid sinus compression; (vi) normal anatomy and function at rest and during stress assessed by the cardiac magnetic resonance and normal electrophysiological study (EPS); (vii) normal ultrasound Doppler of the carotid and vertebral arteries; (viii) able to read, understand and sign the informed consent form, and attend clinic visits and exams. The patients that presented any of the subsequent criteria were excluded: (i) pregnancy; (ii) valvular disease with significant adverse sequelae; (iii) unstable angina, myocardial infarction, transient ischemic attack or stroke within the 6 months before the procedure; (iv) renovascular abnormalities; (v) psychiatric disease; (vi) allergy to ionic contrast; (vii) the inability to be monitored clinically after the procedure; (viii) a known addiction to drugs or alcohol that affects the intellect; (ix) a serious health condition that, in the investigator opinion's, may adversely affect the safety and/or efficacy of the participant or the study; (x) congestive heart failure presenting functional class II to IV symptoms according to New York Heart Association classification.

The 18 subjects underwent EPS. The primary goal of this study evaluated if carotid sinus stimulation (CSS) can provoke changes in the BP and HR leading to low output symptoms. The procedures were performed in the catheterization laboratory with direct visualization using fluoroscopy and radiopaque contrast. The patients were pretreated with diazepam or midazolam under the supervision of an anesthesiologist. At the end of standard EPS perform, a femoral artery was punctured and a short $6 \mathrm{~F}$ sheath (St. Jude Medical, St. Paul, Minnesota, USA) was placed into this vessel, allowing introduce and position the quadripolar dirigible Livewire ${ }^{\text {mix }}$ catheter (St. Jude Medical, St. Paul, Minnesota, USA) with a tip electrode of $2 \mathrm{~mm}$, and other electrodes of $1 \mathrm{~mm}$, alternetaly into the carotid arteries, as close as possible to the carotid sinus on both sides, under fluoroscopic guidance. Unipolar stimulation was performed from the tip of the catheter to target the carotid sinus. The cycle length was set at $200 \mathrm{~ms}$ (300 ppm),

Correspondence to: Márcio Galindo Kiuchi, MD, MPH, Rua Cel. Moreira César, 138 - Centro, São Gonçalo - Rio de Janeiro - Brazil; Tel/Fax: +55 (21) 26047744; E-mail: marciokiuchi@gmail.com

Received: November 01, 2016; Accepted: November 09, 2016; Published: November 14, 2016 
pacing using the maximum-output voltage at $20 \mathrm{~V}$, current of $8 \mathrm{~mA}$, using a pulse amplitude of $2 \mathrm{~ms}$, during $90 \mathrm{~s}$. The EP-Tracer system (Schwarzer Cardiotek GmbH, Im Zukunftspark 3, 74076 Heilbronn, Germany) was used to record and monitor the cardiac rhythm and HR throughout the procedure. And the DX2021 monitor system (Dixtal Biomédica Indústria e Comércio LTDA., SP, Brazil) was used to register the changes in invasive systolic $\mathrm{BP}$ during the procedure. After the stimulation, we waited for the BP and HR to return to baseline values. The patients remained hospitalized in the ward for $24 \mathrm{~h}$ after the procedure.

The results are expressed as a mean and standard deviation for normally distributed data and as median with interquartile range otherwise. All statistical tests were two-sided. Comparisons between

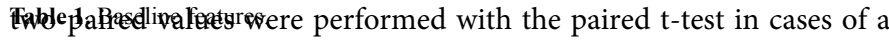

\begin{tabular}{|l|c|}
\hline $\mathrm{N}$ & 18 \\
\hline Age, years & $61.8 \pm 12.4$ \\
\hline Body mass index, $\mathrm{kg} / \mathrm{m}^{2}$ & $26.5 \pm 2.1$ \\
\hline Male gender $(\%)$ & $13(72 \%)$ \\
\hline White ethnicity $(\%)$ & $14(78 \%)$ \\
\hline Uncontrolled hypertension & $18(100 \%)$ \\
\hline Type 2 Diabetes Mellitus (\%) & $6(33 \%)$ \\
\hline Coronary artery disease & $3(17 \%)$ \\
\hline Pre-syncope symptoms & $18(100 \%)$ \\
\hline Normal carotid sinus compression & $18(100 \%)$ \\
\hline Negative tilt-table test & $18(100 \%)$ \\
\hline Normal electrophysiological study & $18(100 \%)$ \\
\hline Normal cardiac magnetic resonance & $18(100 \%)$ \\
\hline Mean 24-hour systolic/diastolic ABPM, mmHg & $142.0 \pm 7.3 / 95.2 \pm 3.9$ \\
\hline Min - ave - max HR at 24-hour-Holter monitoring, bpm & $48.6-73.2-129.5$ \\
\hline Creatinine, mg/dL & $0.72 \pm 0.15$ \\
\hline eGFR, mL/min/1.73 m ${ }^{2}$ (CKD-EPI) & $91.3 \pm 15.4$ \\
\hline Albumin:creatine ratio mg/g & $18.2 \pm 7.5$ \\
\hline Antihypertensive agents & \\
\hline ACEI/ARB & $18(100 \%)$ \\
\hline Diuretics & $18(100 \%)$ \\
\hline DHP Ca ${ }^{++}$channel blockers & $18(100 \%)$ \\
\hline$\beta$-blockers & $11(61 \%)$ \\
\hline Spironolactone & $9(50 \%)$ \\
\hline
\end{tabular}

The values are presented as mean $\pm \mathrm{SD}$ or $\%$; $\mathrm{ABPM}$, ambulatory blood pressure measurements; ACEI, receptor inhibitor of angiotensin converting enzyme; ARB, angiotensin receptor blocker; Ave, average; DHP, dihydropyridyne; eGFR, estimated glomerular filtration rate; HR, heart rate; Min, minimum; Max, maximum; N, number of patients
Gaussian distribution and by the Wilcoxon test otherwise. Comparisons between more than two-paired values were made by repeatedmeasures analysis of variance or by Kruskal-Wallis analysis of variance as appropriate, complemented by a post-hoc test. Categorical variables were compared with Fisher's exact test. A P-value $<0.05$ was considered significant. All statistical analyses were performed using the program Graphpad Prism v 7.0 (Graphpad Software, La Jolla, CA, USA).

The general features of both groups of patients are listed in Table 1. According to Figure $1 \mathrm{~A}$, the right CSS provoked a decrease in the systolic BP from $162.9 \pm 9.1 \mathrm{mmHg}$ at baseline to $129.6 \pm 5.9 \mathrm{mmHg}$ during stimulation $(\mathrm{P}<0.0001)$ and the diastolic $\mathrm{BP}$ reduced from $111.4 \pm 9.7$ to $82.9 \pm 3.9 \mathrm{mmHg}(\mathrm{P}<0.0001)$, as shown in Figure $1 \mathrm{~A}$. The left CSS led to a decrease from $162.4 \pm 8.0 \mathrm{mmHg}$ at baseline to $150.5 \pm 10.9 \mathrm{mmHg}$ during stimulation $(\mathrm{P}<0.0001)$ and the diastolic $\mathrm{BP}$ reduced from $110.6 \pm 10.0$ to $86.0 \pm 4.2 \mathrm{mmHg}(\mathrm{P}<0.0001)$, as shown in Figure 1B. The comparisons between the variation $(\Delta)$ of the systolic BP between the right CSS $(-33.3 \pm 6.4 \mathrm{mmHg})$ and left CSS $(-11.9 \pm 6.1 \mathrm{mmHg})$ was significant $(\mathrm{P}<0.0001)$. However, the same comparisons between the $\Delta$ of the diastolic BP between the right CSS $(-28.4 \pm 9.2 \mathrm{mmHg})$ and left CSS $(-24.6 \pm 11.3 \mathrm{mmHg})$ were not different $(\mathrm{P}=0.2703)$, as observed in Figure 1C. The right CSS provoked a decrease at $\mathrm{HR}$ from $81.7 \pm 6.9 \mathrm{bpm}$ at baseline to $56.2 \pm 5.4 \mathrm{bpm}$ (Figure 2A) during stimulation $(\mathrm{P}<0.0001)$, and the left CSS provoked a reduction at $\mathrm{HR}$ from $82.1 \pm 7.1$ to $69.4 \pm 6.3 \mathrm{bpm}$ (Figure $2 \mathrm{~B}$ ). The comparison between the $\Delta$ of the HR between the right CSS $(-25.5 \pm 8.1$ bpm) and the left CSS $(-12.6 \pm 4.3 \mathrm{bpm})$ was important $(\mathrm{P}<0.0001)$, as shown in Figure 2C.

In conclusion, our study shows that an acute fall in invasive systolic/ diastolic BP and HR are observed during the right and the left CSS. This effect is more intense to the right than the left CSS regarding the fall in the invasive systolic BP and the HR. These findings suggest the importance of the vagal tone as one of the mechanisms to suppress hypertension.

\section{Conflict of interest}

None declared.

\section{Funding} Brazil.

This study was funded by Pacemed (US \$200,000), Rio de Janeiro,
(A)

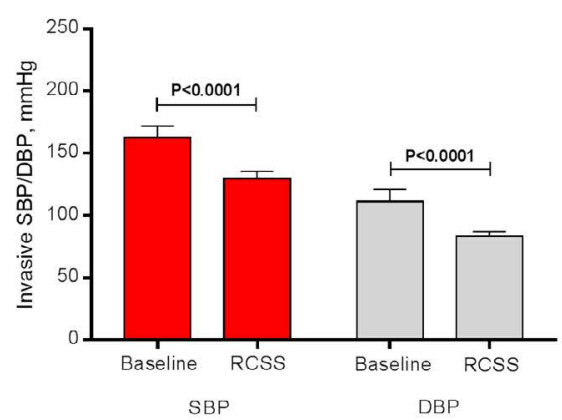

(B)

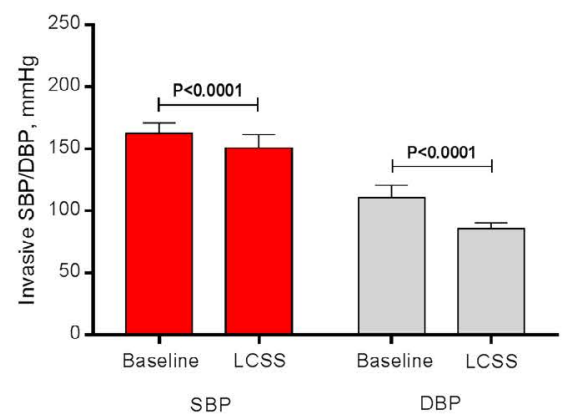

(C)

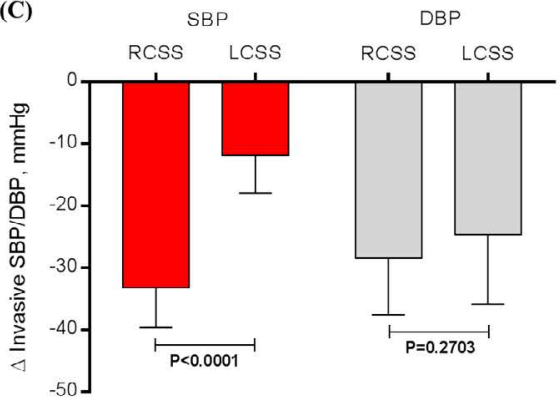

Figure 1. Effects of the right (A) and the left (B) CSS in the invasive systolic and diastolic blood pressure, as well as, in the comparisons between the variation ( $\Delta$ ) of the systolic and the $\Delta$ of the diastolic (C) during the right and the left CSS. CSS, carotid sinus stimulation; N=18. 
(A)

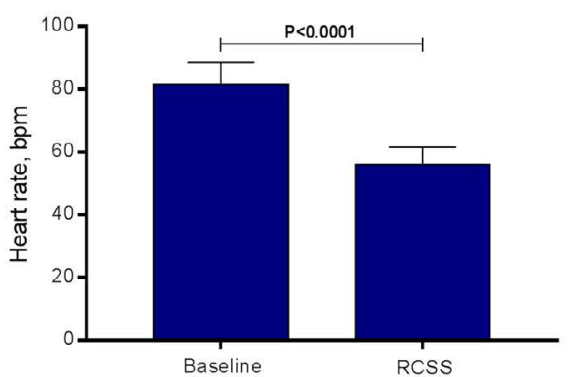

(B)

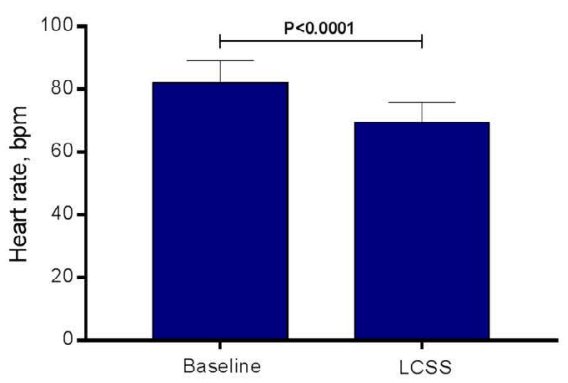

(C)

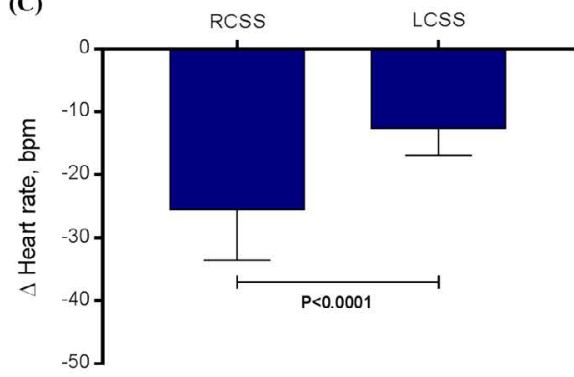

Figure 2. Effects of the right (A) and the left (B) CSS in the heart rate, as well as, in the comparison between the variation ( $\Delta$ ) of heart rate (C) during the right and the left CSS. CSS, carotid sinus stimulation; $\mathrm{N}=18$.

\section{Acknowledgements}

The authors are grateful to all participants included in this study. The authors also thank Pacemed for stimulating the development of this study and for providing technical support.

\section{References}

1. Scheffers IJ, Kroon AA, Schmidli J, Jordan J, Tordoir JJ, et al. (2010) Novel baroreflex activation therapy in resistant hypertension: results of a European multi-center feasibility study. $J$ Am Coll Cardiol 56: 1254-1258. [Crossref]

2. Bakris GL, Nadim MK, Haller H, Lovett EG, Schafer JE, et al. (2012) Baroreflex activation therapy provides durable benefit in patients with resistant hypertension: results of long-term follow-up in the Rheos Pivotal Trial. J Am Soc Hypertens 6: 152158. [Crossref]

3. Hoppe UC, Brandt MC, Wachter R, Beige J, Rump LC, et al. (2012) Minimally invasive system for baroreflex activation therapy chronically lowers blood pressure with pacemaker-like safety profile: results from the Barostim neo trial. $J \mathrm{Am} \mathrm{Soc}$ Hypertens 6: 270-276. [Crossref]

4. Wallbach M1, Halbach M, Reuter H, Passauer J, Lüders S, et al. (2016) Baroreflex activation therapy in patients with prior renal denervation. J Hypertens 34: 1630-1638. [Crossref]

5. Levey AS, Stevens LA, Schmid CH, Zhang YL, CKD-EPI (Chronic Kidney Disease Epidemiology Collaboration), et al. (2009): A new equation to estimate glomerular filtration rate. Ann Intern Med 150: 604-612. [Crossref]

Copyright: $₫ 2016$ Kiuchi MG. This is an open-access article distributed under the terms of the Creative Commons Attribution License, which permits unrestricted use, distribution, and reproduction in any medium, provided the original author and source are credited. 